is also a known association between AS and conduction disturbances and aortic regurgitation. Comparative analyses in the same setting of the strengths of these associations with AS, PsA and USpA are scarce.

Objectives: To assess the strengths of the associations of different SpA-related comorbidities with a diagnosis of AS, PsA and USpA.

Methods: All patients, aged $\geq 18$ years, with AS ( $n=3884)$, PsA $(n=8706)$ and uSpA ( $n=2665$ ) were identified 2001 through 2005, according to specified ICD codes from the Swedish Patient Register. The register contains diagnoses from all visits in inpatient and non-primary outpatient care. Each patient was matched by year of birth, sex and county to five general population (GP) controls identified in the Population Register. Occurrence of SpA-related comorbidities prior to 1 January 2006 were also retrieved from the Swedish Patient Register. Number and proportion of cases ( $\mathrm{n}(\%)$ ) with a recorded SpA-related comorbidity prior to 1 January 2006 and corresponding prevalence ratio (PR) with 95\% confidence interval $(\mathrm{Cl})$ were calculated, overall and stratified for sex.

Results: PRs for SpA-related comorbidities were significantly elevated in all SpA subtypes compared to their matched GP controls (Table). PRs were substantially elevated in AS, intermediately elevated in USpA, whereas only moderately increased in PsA. The results were similar in the sex-stratified analyses (not shown).

Conclusions: The strongest associations of SpA-related comorbidities were seen with AS, closely followed by uSpA, compatible with a substantial shared etiology and phenotypical expression for patients given these two diagnoses, whereas the associations between PsA and the SpA-related comorbidities were considerably weaker.

Disclosure of Interest: None declared

DOI: 10.1136/annrheumdis-2017-eular.1255

\section{OP0241 ERAP1 IS A SUSCEPTIBILITY FACTOR FOR EARLY AXIAL SPONDYLOARTHRITIS MEETING THE ASAS CLASSIFICATION CRITERIA: RESULTS FROM THE DESIR AND SPACE COHORTS}

A. de Koning ${ }^{1}$, M. Hameetman ${ }^{1}$, C. Micelli ${ }^{2}$, M. Dougados ${ }^{3}$, D. van der Heijde ${ }^{1}$, F. Kurreeman ${ }^{1}$, F.A. van Gaalen ${ }^{1} .{ }^{1}$ LUMC, Leiden, Netherlands; ${ }^{2}$ Hôpital Bicêtre; ${ }^{3}$ Hôpital Cochin, Paris, France

Background: Genetic research in axSpA is performed in patients with longstanding ankylosing spondylitis (AS). Early axial spondyloarthritis (axSpA) is a diverse patient group in which a minority of the patients have AS. Therefore, it is not known if recently identified genetic risk factors for AS are also risk factors for early axSpA.

Objectives: To evaluate if ERAP1 and HLA-B*4001 are susceptibility factors for early axSpA.

Methods: Patients with early axSpA meeting the ASAS classification criteria from the SPACE cohort (inclusion criteria: back pain for $\geq 3$ months, $\leq 2$ years, onset $<45$ years) and the DESIR cohort (inclusion criteria: inflammatory back pain for $>3$ months, $<3$ years, age $<50$ ) were typed for two well established AS genetic risk factors: ERAP1 SNPs rs30187 (susceptibility), rs17482078 (protective) and rs 10050860 (neutral) and HLA-B*4001. Analysis of weaker AS risk factors was limited by sample size. For ERAP1, genotyped Dutch healthy controls $(n=1085)$ and published French controls ${ }^{1}$ were used as controls. For HLA-B*4001 healthy blood bank donors from the Netherlands $(n=5584)$ and France $(n=10177)$ were used as controls.

Results: In 486 DESIR patients (mean age 32.5 (SD 8.6); 50\% male; $84 \%$ HLA-B27+; 18\% X-SI+) and 144 SPACE patients (mean age 29.5 (SD 8); 51\% male; $88 \%$ HLA-B27+; 24\% X-SI+) ERAP1 SNP rs30187 was more common than in controls (OR 1.2, $\mathrm{p}=0.01$ in meta-analysis; table 1). rs 17482078 and rs10050860 were negatively associated with early axSpA (both $0.7(0.6-0.9)$ $p<0.01$ in meta-analysis). Although both cohorts were sufficiently powered, HLA-B*4001 was not positively associated with early axSpA (OR $0.6(0.4-0.9)$ in meta-analysis)

\begin{tabular}{|c|c|c|c|c|c|c|c|c|}
\hline & & $\begin{array}{l}\text { SPACE } \\
n=144\end{array}$ & $\begin{array}{c}\text { Dutch controls } \\
n=1085\end{array}$ & & $\begin{array}{l}\text { DESIR } \\
n=486\end{array}$ & $\begin{array}{c}\text { French controls" } \\
n=384\end{array}$ & & Meta-analysis \\
\hline & Risk allele & MAF & MAF & OR $(95 \%(\mathrm{Cl})$ & & MAF & OR (95\% (I) & OR (95\% CI) \\
\hline rs 530187 & & 0.38 & 0.32 & $1.2(1.0-1.6)$ & 0.41 & 0.37 & $1.2(0.9-1.5)$ & $1.2(1.0-1.4) p=0.01$ \\
\hline rs174882078 & 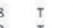 & 0.14 & 0.21 & $0.6(0.5-0.9)$ & 0.18 & 0.22 & $0.8(0.6-1.0)$ & $0.7(0.6-0.9) p<0.001$ \\
\hline is 10050860 & $T$ & 0.15 & 0.22 & $0.6(0.5-0.9)$ & 0.18 & 0.23 & $0.8(0.6-1.0)$ & $0.7(0.6-0.9)_{p}<0.001$ \\
\hline
\end{tabular}

Conclusions: ERAP1 rs30187 is a genetic risk factor for early axSpA. To our knowledge this is the first report of genetic risk factor research in early axSpA patients meeting the ASAS criteria. Larger cohorts are needed to study additional AS risk factors.

References:

[1] Kadi A, Izac B, Said-Nahal R, Leboime A, Van Praet L, de Vlam K, et al. Investigating the genetic association between ERAP1 and spondyloarthritis. Ann Rheum Dis. 2013;72(4):608-13.

Disclosure of Interest: None declared

DOI: 10.1136/annrheumdis-2017-eular.5955

\section{OP0242 HEALTH-RELATED QUALITY OF LIFE IMPROVED ONLY SLIGHTLY ALTHOUGH DISEASE ACTIVITY DECREASED OVER 3 YEARS IN EARLY AXIAL SPONDYLOARTHRITIS: 442 PATIENTS FROM THE DESIR COHORT}

D. Puyraimond-Zemmour ${ }^{1,2}$, C. Desthieux ${ }^{1,2}$, A. Ruyssen-Witrand ${ }^{3}$,

B. Fautrel ${ }^{1,2}$, L. Gossec ${ }^{1,2}$. ${ }^{1}$ Paris 06 university, Pierre and Marie Curie; ${ }^{2}$ Pitie

Salpetriere Hospital, Paris; ${ }^{3} \mathrm{CHU}$ de Toulouse, Hôpital Pierre-Paul Riquet,

Toulouse, France

Background: Current guidelines emphasize Health-related Quality of life (HRQoL) as a priority in health care but the link with disease activity is incomplete.

Objectives: The objective was to describe in patients with early axial spondyloarthritis (axSpA) the evolution of SF36 and of ASDAS-CRP over a 3 years follow up.

Methods: DESIR is an ongoing prospective, multicenter, longitudinal, observational French cohort (1). Patients had inflammatory back pain of more than 3 months and less than 3 years suggestive of axSpA. For our analysis, only patients with no missing data for MCS and PCS SF36 values during the first 3 years were included. HRQoL was assessed by the SF36 questionnaire and its 2 subscales (scored $0-100$ with higher scores indicating better status): the physical composite score (PCS) including physical function, physical role, bodily pain and general health $(\mathrm{GH})$ and the mental composite score (MCS) including mental health $(\mathrm{MH})$, emotional role, social function and vitality. Disease activity was assessed by the ASDAS-CRP. Each outcome was assessed at baseline, 6 months, 12 months, 24 months and 36 months. The mean and median outcomes were calculated at each time-point and comparisons used ANOVA on repeated data. Baseline predictive factors associated with worse SF36 scores (lower half of mean values over 3 years) were identified by univariate then multivariate logistic regression, among the baseline variables age, gender, educational level, work status, smoking, HLAB27, radiological sacroiliitis, extra-articular manifestations (uveitis, psoriasis, inflammatory bowel disease) and ASDAS-CRP.

Results: In all, 442 patients were analyzed: at baseline, mean age was $34.2 \pm 8.6$ years; mean disease duration was $18.6 \pm 10.7$ months; $239(54 \%)$ were females, $274(62 \%)$ had HLAB27 and $124(28 \%)$ had radiological sacroiliitis. At baseline, mean PCS, MCS and ASDAS-CRP were respectively 40.5 $\pm 9.2,40.4 \pm 11.0$ and 2.6 \pm 1.0 . Over 3 years, although mean ASDAS-CRP improved, HRQoL improved only slightly (Figure). All the SF36 subdomains improved (all $p<0.001$ ), though $\mathrm{GH}$ and $\mathrm{MH}$ improved less (data not shown). In univariate analysis, lower educational level, not being in a white collar job, younger age and higher ASDAS-CRP were significantly associated to lower HRQoL over 3 years. In multivariate analysis, only higher baseline ASDAS-CRP (odds ratio=0.49 [95\% confidence interval 0.29; 0.79]) was associated to lower HRQoL.

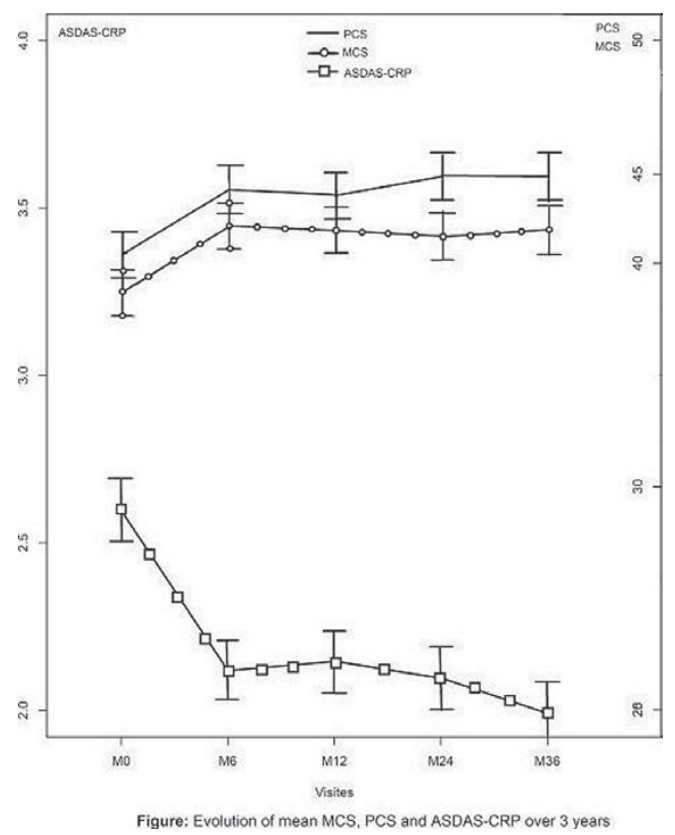

Conclusions: In early axSpA, HRQoL was affected similarly for the physical and mental components and only slightly improved over 3 years. In contrast, ASDASCRP improved substantially. However, ASDAS-CRP was a stronger driver of $\mathrm{HRQ}$ oL than demographic characteristics. The drivers of HRQoL in inflammatory rheumatic disorders should be further explored.

References:

[1] Dougados M. et al. Clinical presentation of patients suffering from recent onset chronic inflammatory back pain suggestive of spondyloarthritis: The DESIR cohort.Joint Bone Spine, 2015;82(5):345-51.

Disclosure of Interest: None declared

DOI: 10.1136/annrheumdis-2017-eular.4806 